\title{
The Dual Nature of Matter and the Uncertainty Principle Led the Quantum Theory to Wave Mechanics
}

\author{
Dr.(Prof.) V.C.A. NAIR* \\ Educational Physicist and Research Guide for Physics at JJT University, Rajasthan-333001, India
}

\begin{abstract}
The paper begins with a brief account of classical theory of radiation and its failure to explain black body radiation. It is followed by the introduction of Quantum Theory of radiation by Max Planck. The dual nature of matter by de Broglie has been extensively dealt with. The uncertainty principle of Heisenberg has been critically discussed by many eminent authors who finally concluded it as one of the unavoidable mysteries of nature. As a picture speaks thousand words and as face is the index of the mind, the author has added pictures of eminent physicists at appropriate places. The subject matter of the paper will form a good text for any graduate students of Physics.
\end{abstract}

Keywords: Classical theory, Dual nature, Photon, Planck's constant, Quantum Theory, Schrodinger equation, Ultraviolet catastrophe, Uncertainty principle, Wave mechanics.

\section{INTODUCTION}

The paper is classified into four parts

1. Failure of the Classical Theory and introduction of the Quantum Theory by Max Planck.

2. De Broglie's theory of Matter Waves.

3. Development of the Quantum Theory in the form of Wave Mechanics and Quantum Mechanics by

Erwin Schrodinger and Werner Heisenberg respectively.

4 . Heisenberg's Principle of Indeterminacy or Uncertainty Principle

In the review of literature that follows, each of the above has been extensively dealt with. Generally one discards old things. But, it is the old thing that makes things bright and start thinking about the events of the past. There are very few authors who have attempted a Research Paper on a topic of this kind and hence for any subject matter one has to depend on classical books written by eminent authors among which J.B. Rajam's book on Atomic Physics tops the list and a major part of my paper is from that and I have acknowledged the same at the end. For the benefit of readers, I may, however, mention one or two research papers:

1."De Broglie Waves as the "Bridge" of becoming between Quantum Theory and Relativity" by Ruth E. Kastner Foundations of Physics Group, University of Maryland, College Park, USA, 7/5/2011.

2. "The Quantum Theory of Waves and Particles" by John D. Norton, Dept. of History and Philosophy of Science, University of Pittsburg, $14^{\text {th }}$ April 2017.

3."On the Theory of Quanta" by Louis Victor de Broglie", Translation by A.F. Kracklauer, 2004

As a fourth one, one may go through De Broglie's Nobel Lecture itself, a part of which has been referred to in my paper. The subject matter given in the above papers remains the same and any attempt in making anything new ultimately ends up with putting old wine in new bottle. However, I am sure (and I hope) the readers will appreciate my presentation. Some of the diagrams in the paper are drawn by author himself.

\section{REVIEW OF LITERATURE \\ II FAILURE OF THE CLASSICAL THEORY AND INTRODUCTION OF THE QUANTUM THEORY BY MAX PLANCK}

2.1 The Origin: The Corpuscular theory of light as suggested by the English mathematician, astronomer, theologian and physicist, Sir Isaac Newton (Fig.3 a) could not explain certain phenomena like Refraction and Diffraction. It was later challenged by Huygens and Fresnel by their famous wave theory of light. By this theory they could explain almost all phenomena associated with light and radiation. Even the wave theory of light broke down because according to that the velocity of propagation of light through a medium is given by $v=\sqrt{ }\left(\frac{\mathrm{E}}{\rho}\right)$ where $\mathrm{v}$ is the velocity of light, $\mathrm{E}$ the 
elastic modulus of the medium and $\rho$ its density. As $\mathrm{v}$ is very high of value $3 \times 10^{8} \mathrm{~ms}^{-1}$, the elasticity of the medium comes out to be very high and hence becomes contradictory. Further, this led to the conclusion that the medium is a most rigid elastic solid. At this juncture, the Scottish mathematical physicist, James Clerk Maxwell (Fig.3 b) came forward with his classical Electromagnetic Wave Theory of Light. He started with an original and novel idea of A 'Displacement Current'. According to this new concept, light or radiation is considered as the result of rapidly alternating displacement currents in the medium which give rise to magnetic fields, similar to those with conduction currents. The two fields, the electric and magnetic inseparably associated, the one varying proportionately with the other and the variation of one giving rise to the other, urge each other with a finite velocity, viz. that of light and given by $\frac{1}{\sqrt{\left(\varepsilon_{0} \mu_{0}\right)}}$, where $\varepsilon_{0}$ and $\mu_{0}$ are specific inductive capacity and permeability respectively of the medium. It did not much involve the elasticity of the medium and Maxwell's electromagnetic theory of light was fully accepted.

2.2 The Failure: Two of the basic results of the electromagnetic theory are:

i) Radiation of energy is associated with a moving charge whether it is accelerated or decelerated. The radiation of energy takes place in the form of electromagnetic pulse or wave train as sound waves originate from a sounding body such as a tuning fork. The rate of loss of energy $E$ due to radiation from a charge ' $q$ ' moving with an acceleration ' $a$ ' is given by

$$
\frac{\mathrm{dE}}{\mathrm{dt}}=\left(\frac{2 q^{2} a^{2}}{3 \mathrm{c}^{3}}\right) \text { where 'c' is the velocity of light }
$$

ii)Continuity of action: Since the electric and magnetic fields vary continuously with time, the flow of energy in an electromagnetic wave should be continuous over the entire wave front. In short, the radiation of energy must take place in a continuous fashion.

These results of the electromagnetic theory of Maxwell given above made the nuclear atom model given by Rutherford unstable. The nuclear atom model put forward by Rutherford consists of a central nucleus around which are certain number of electrons assumed stationary and arranged around the nucleus in proper order, Consider for simplicity a two electron atom as in Fig.1 situated diametrically opposite to the nucleus and distance 'r' from it, and ' $\mathrm{e}$ ' the electronic charge. The force of attraction between the positively charged nucleus and the negatively charged electron and the force of repulsion between the two electrons can be worked and given by avoiding the constants that might arise in any system of units.

$$
\begin{aligned}
& \text { Force of attraction }=\frac{2 \mathrm{e} e}{\mathrm{r}^{2}}=\frac{2 \mathrm{e}^{2}}{\mathrm{r}^{2}} \\
& \text { Force of repulsion }=\frac{\mathrm{e} e}{(2 \mathrm{r})^{2}}=\frac{\mathrm{e}^{2}}{4 \mathrm{r}^{2}}
\end{aligned}
$$
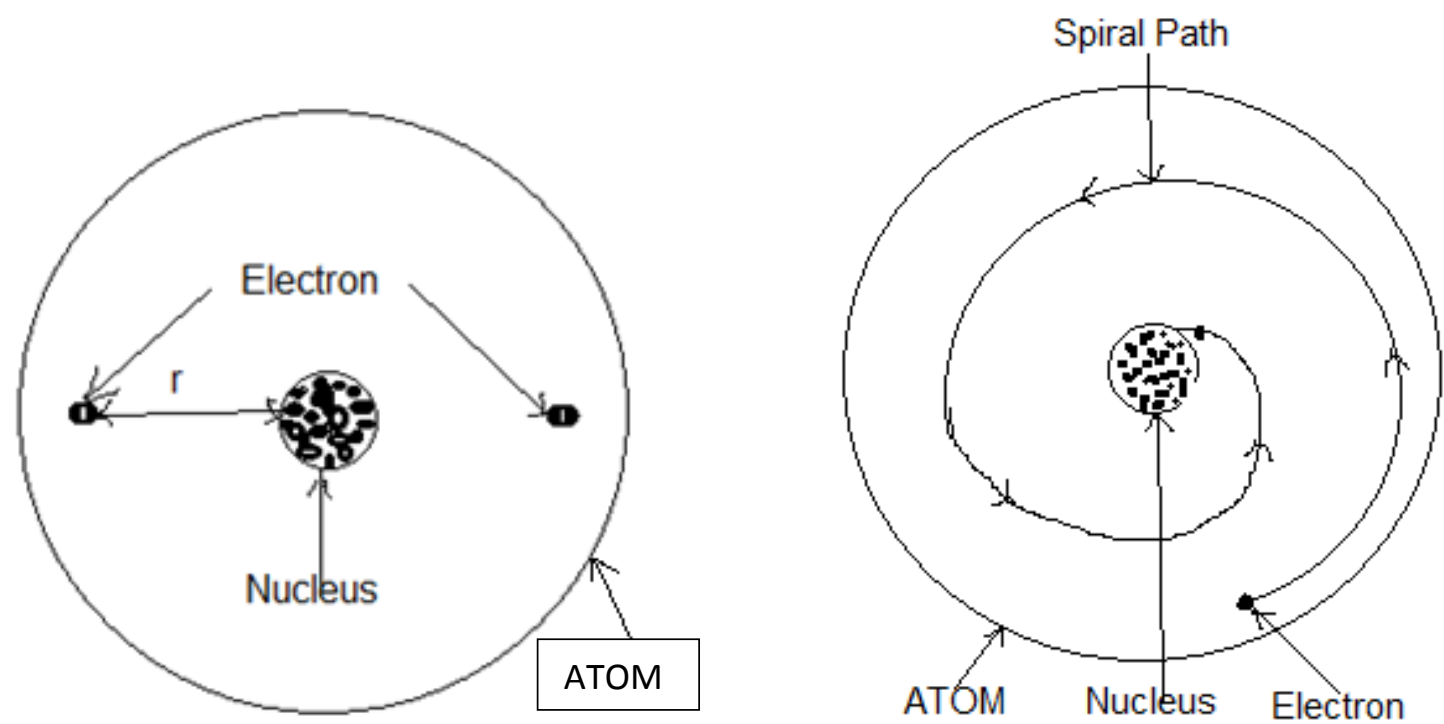

Fig,1 Rutherford's Atom Model

Fig,2 Imagination of the electron spiraling towards the nucleus

It is seen that the force of attraction is 8 times the force of repulsion and hence the electrons which are assumed to be stationary, will fall inside the nucleus due to the force of attraction thereby losing the stability of the atom. 


\section{International Advanced Research Journal in Science, Engineering and Technology}

ISO 3297:2007 Certified

Vol. 4, Issue 12, December 2017

In order to overcome this difficulty, Rutherford assumed that the electrons are not stationary but move around the nucleus in certain orbits similar to planets that move around the Sun. This suggestion was well received by scientists. But, according to the classical electromagnetic theory of Maxwell, a moving electron must radiate energy in a continuous fashion in which case, as no energy is derived from outside sources, the electron in the atom must be continuously losing energy and must become weak and fall inside the nucleus by traversing a spiral path. (Fig.2) The stability of the atom is again lost. This was one of the major failures of the classical electromagnetic theory.

The second major failure was in the explanation of the 'black body radiation' put forward by many and some ten stalwarts among whom are shown in Fig.3 in a chronological order of their existence due to which Albert Einstein (Fig. 3j) happens to occupy the last place. The first among the pictures is of Sir Isaac Newton (Fig.3a). Newton was, perhaps, the only scientist among the few in his times to assign the word 'radiation' for both 'light' and 'heat' His main failure was in the explanation regarding the nature of these two especially the first one and the second one taken up as 'thermal radiation' two centuries later by other scientists. At this juncture I would like to quote what Bergmann [1, preface, p.v] has said in in the preface of his classical book, Basic Theories of Physics-Heat and Quanta, "Of all the classical theories of physics, the theory of heat is undoubtedly the most difficult and perhaps because of its intellectual challenge to many of us the most fascinating" It is the investigations done by physicists on thermal radiation that have revolutionized the entire Physics which gave birth to the Quantum and started a new era in Modern Physics.

As early as 1859 the Prussian-German physicist, Kirchhoff Gustav (Fig.3 c) was the first scientist to study quantitatively the behavior of a black body. A black body is a surface which will receive all the heat given to it and when allowed to cool, will radiate all the heat received by it. One may even call such a body as a perfectly black body. Kirchhoff asserted that the radiation from a black body depends only on the temperature and not on the nature or on the material of the body.

I shall arrive at the law from Roberts [9. P.381-3]. Consider an isotropic body at temperature $\mathrm{T}$ emits radiation of energy $Q$ into vacuum in unit time per unit area and if the wavelength lies between $\lambda$ and $\lambda+d \lambda$. If $a_{\lambda}$ is the absorptive power of the surface, then the fraction of the energy absorbed is: $a_{\lambda} \mathrm{dQ}$. The remaining

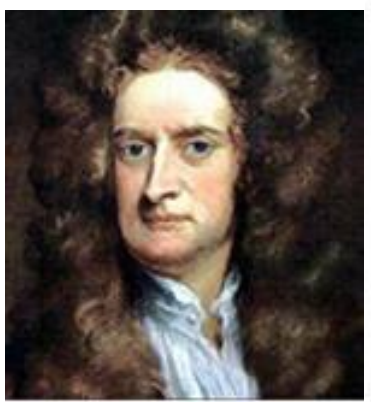

(a) Sir Isaac Newton (1642-1727)

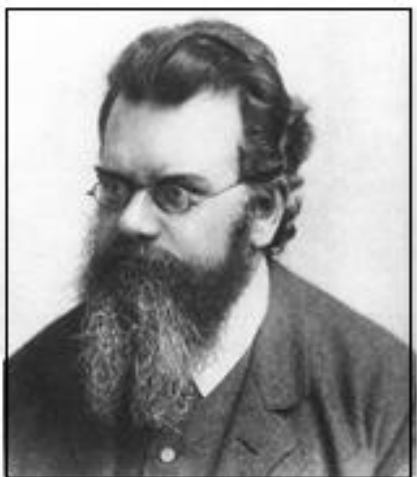

(e) Ludwig Boltzmann(1844-1906)

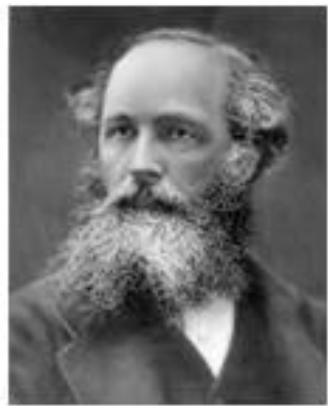

(b) James Clerk Maxwell (1831-1879)

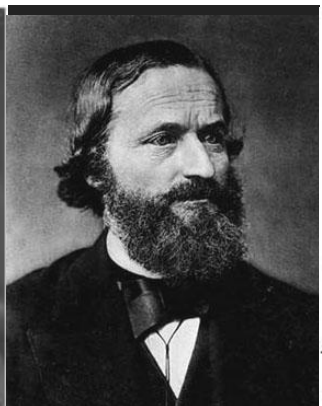

(c) Kirchhoff Gustav (1824-1887)

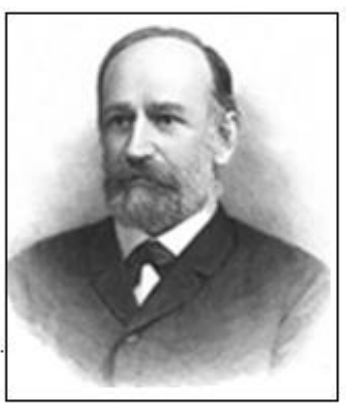

(d) Stefan Josef (1835-1893)

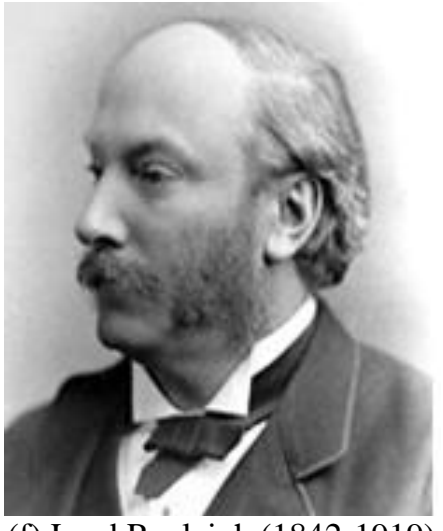

(f) Lord Rayleigh (1842-1919)

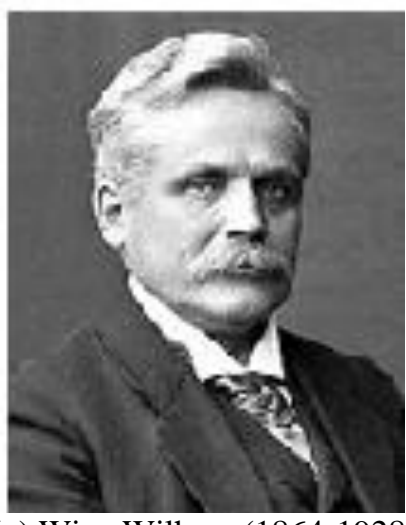

(g) Wien Wilhem (1864-1928)

$\left(1-a_{\lambda}\right) \mathrm{dQ}$ is either reflected or transmitted. If $\mathrm{e}_{\lambda}$ is the emissive power of the body, the quantity of energy of the given wavelength emitted by the body per unit area per second is $e_{\lambda} d \lambda$. In order to maintain equilibrium in the enclosure, the amount of energy absorbed must equal the amount of energy emitted and hence:

$$
a_{\lambda} \mathrm{dQ}=\mathrm{e}_{\lambda} \mathrm{d} \lambda \text {. }
$$




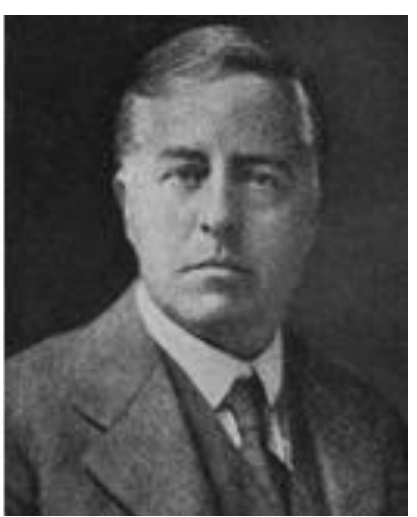

(h) James Jeans (1877-1946)

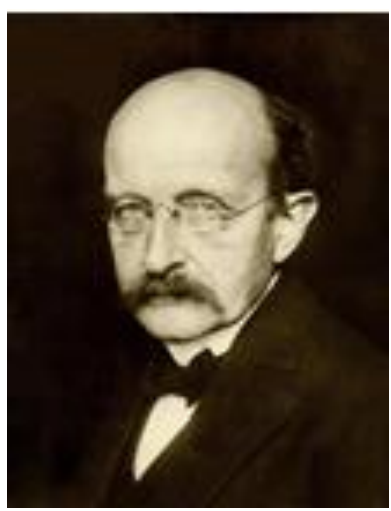

(i) Max Planck (1858-1947)

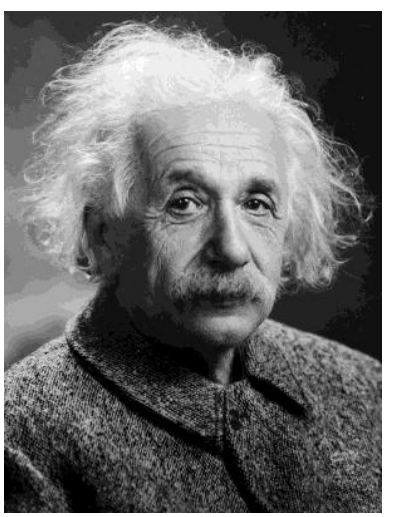

(j) Albert Einstein (1879-1955)

Fig. 3 The 10 stalwarts of Thermal Radiation in a chronological order of their existence

$\therefore \frac{\mathrm{dQ}}{\mathrm{d} \lambda}=\frac{e_{\lambda}}{a_{\lambda}}$ which depends only on the temperature and hence

$$
\frac{e_{\lambda}}{a_{\lambda}}=\text { constant for a given temperature }
$$

That is, the "Ratio of emissive power to the absorptive power for radiation of a given wavelength is the same for all bodies at the same temperature" which is Kirchhoff's law

In the year 1884 the physicist, mathematician and poet, Stefan Josef (Fig. 3 d) and the Austrian physicist and philosopher, Boltzmann (Fig. 3 e) came forward and applying the idea of pressure exerted by electromagnetic radiation and also based on thermodynamic principles, arrived at a conclusion that the energy E radiated by a black body is directly proportional to the fourth power of the Absolute temperature and the same is expressed by

$$
\mathrm{E}=\sigma \mathrm{T}^{4}
$$

Where $\sigma$ is the famous Stefan-Boltzmann constant the value of which is $5.67 \times 10^{-8} \mathrm{~W}^{-2} \mathrm{~K}^{-4}$.

In the year 1893, the German physicist Wilhem Wien (Fig.3 g) considering the adiabatic expansion in a container arrived at an expression for the energy distribution of black body radiation as

$$
\mathrm{dE}=\mathrm{K} \lambda^{-5} \mathrm{e}^{-\left(\frac{\mathrm{a}}{\lambda \mathrm{T}}\right)} \mathrm{d} \lambda
$$

where $\mathrm{K}$ and a are constants. He further showed that the product $\lambda \mathrm{T}$ for a particular radiation is constant the value of which is $2.898 \times 10^{-3} \mathrm{~m}^{0} \mathrm{~K}$.

In the year 1900, the English physicist, Lord Rayleigh (Fig.3 f) and another English physicist, astronomer and mathematician, James Jeans (Fig.3 h) struck strictly on the electromagnetic theory of maxwell and asserted that the black body emits radiations of 'Continuously variable' wavelengths from zero to infinity and with this as the basis obtained the following expression for the energy distribution of black body radiation:

$$
\mathrm{dE}=\mathrm{B} \lambda^{-4} \mathrm{~T} \mathrm{e}^{-\left(\frac{\mathrm{a}}{\lambda \mathrm{T}}\right)} \mathrm{d} \lambda
$$

where a and $\mathrm{B}$ are constants.

\subsection{The Ultraviolet Catastrophe:}

These different formulae for the energy distribution of black body radiation obtained on the basis of classical theory led to wrong and absurd conclusions as they did not fit into experimental curves obtained earlier by scientists such as Paschen, Lummer, Pringsheim and others. For example, the energy distribution formula of Wien agreed with region of short wavelengths whereas the formula of Rayleigh and Jeans agreed with the region of long wavelengths. Thus, not a single formula could explain the nature of the experimental curves throughout their wavelength range. This led to the failure of the classical theory because the theoretical derivations were mathematically correct and free from errors, an anomalous situation had to be accepted in the disagreement of theory with experiment unless one assumed the 


\section{International Advanced Research Journal in Science, Engineering and Technology}

ISO 3297:2007 Certified

Vol. 4, Issue 12, December 2017

fundamental assumptions of the classical theory were at fault This is known in classical physics as the "Ultraviolet Catastrophe”. In fact it is the catastrophe of classical physics.

\subsection{Planck's Theory of Quanta:}

The ultraviolet catastrophe led the great German theoretical physicist, Max Planck (Fig.3 i), Dean of the Academy of Sciences at the University of Berlin proposed at the end of the year 1900 and to be exact on 14 December that year a new revolutionary hypothesis known as the "Theory of Quanta" by means of which he was able to derive a correct law of thermal radiation. With the arrival of this theory, the entire classical theory broke down.

The recent advancements in technology, industry, agriculture, medicine and rocketry are due to a small seed sown on 14 December 1900 into the fertile soil of scientific knowledge by Max Planck and, since, carefully cultivated by a whole galaxy of brilliant scientists prominent among whom are Albert Einstein

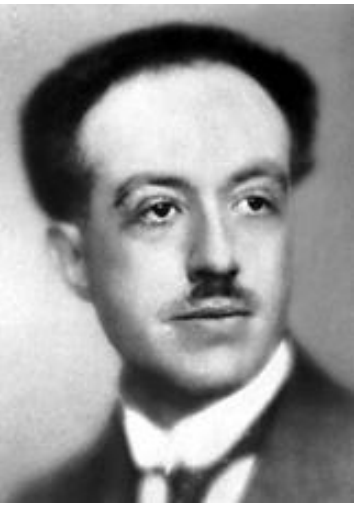

(a)Louis de Broglie (1892-1987)

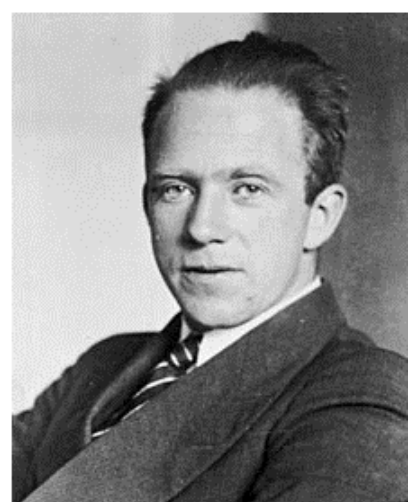

(b) Werner Heisenberg (1901-1976)

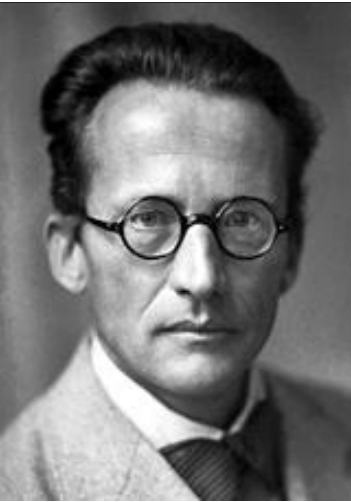

(c) Erwin Schrodinger (1887-1961)

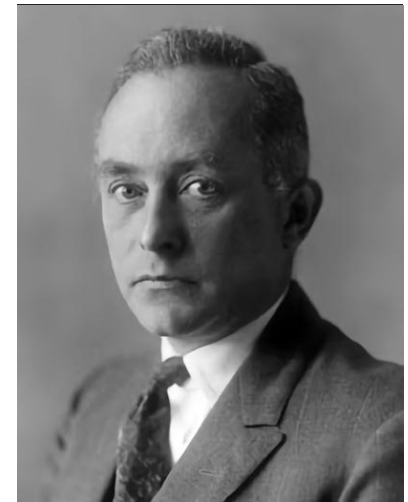

(d) Max Born

(1882-1970)

Fig.4.The stalwarts of Wave Mechanics

(Fig.3j), Louis de Broglie (Fig.4 a), Werner Heisenberg (Fig.4 b), Erwin Schrodinger (Fig.4 c) and Max Born (Fig.4 d) who are considered as the founding fathers of Quantum Physics. Planck argued that the classical ideas of continuity of action might be wrong and proposed instead, that the energy changes could take place only discontinuously and discretely always as integral multiples of a small unit of energy which he called "Quantum". The light quanta of different types of radiation carry different amounts of energy and that the amount of energy of a light quantum is directly proportional to its frequency. Writing $v$ for the frequency of radiation and E the energy of the light quantum, we can express Planck's assumption in the form

$$
\mathrm{E}=\mathrm{h} v
$$

where $\mathrm{h}$ is the constant of proportionality known as the Planck's constant the value of which is $6.62 \times 10^{-34}$ Joule second. The value of this constant is as great to Physics as its magnitude is small. From the unit, 'Joule second (Js)', whether the energy is small or time is small, their product is small leading to something like 'action' and it is the Planck's constant of action and is the smallest action ever known in the universe.

With this as the basis, Planck derived the following expression for the energy distribution of black body radiation.

$$
\mathrm{dE}=\frac{8 \pi \mathrm{hc} \lambda^{-5}}{\left[e^{\frac{\mathrm{hc}}{(\mathrm{k} \lambda \mathrm{T})}}-1\right]} \mathrm{d} \lambda
$$

where $\mathrm{h}$ is the Plank's constant, $\mathrm{c}$ the velocity of light, $\lambda$ the wavelength, $\mathrm{k}$ the Boltzmann constant and T the absolute temperature. The formula can also be written in terms of the frequency $v$ of the radiation following $c=v \lambda$. The formula is in full agreement with the experimental curves (Fig. 5a[13] and Fig.5b [ 9.p.opp.392]) Fig. 5 is from Wikipedia [13] and $5 \mathrm{~b}$ is from Roberts [9, p.opp.392] and appears to be original.

Planck' law given by equation (4) is in perfect agreement with the experimental curves. Further, the earlier radiation laws of Wien and Rayleigh-Jeans can be obtained from this law as particular cases. Thus, for the short wavelength region, $e^{\frac{\mathrm{hc}}{(\mathrm{k \lambda T})}}$ becomes large compared to unity so that Planck's formula reduces to

$$
\mathrm{dE}=8 \pi \mathrm{hc} \lambda^{-5}\left(e^{-\left[\frac{\mathrm{hc}}{(\mathrm{k} \lambda \mathrm{T})}\right]}\right) \mathrm{d} \lambda=\mathrm{K} \lambda^{-5}\left(e^{-\left[\frac{\mathrm{a}}{(\lambda \mathrm{T})}\right]}\right) \mathrm{d} \lambda, \mathrm{K} \text { and a being constants }
$$

which is Wien's law given by equation (2)

For long wavelengths, $\left[e^{\frac{\mathrm{hc}}{(\mathrm{k} \lambda \mathrm{T})}}-1\right]$ becomes nearly equal to $\left[\frac{\mathrm{hc}}{(\mathrm{k} \lambda \mathrm{T})}\right]\left[e^{\frac{\mathrm{hc}}{(\mathrm{k} \lambda \mathrm{T})}}\right]$ so that 


\section{International Advanced Research Journal in Science, Engineering and Technology}

\section{ISO 3297:2007 Certified}

Vol. 4, Issue 12, December 2017

$\mathrm{dE}=8 \pi \mathrm{hc} \lambda^{-5}\left(\frac{\mathrm{k} \lambda \mathrm{T}}{\mathrm{hc}}\right) e^{-\left[\frac{\mathrm{hc}}{(\mathrm{k} \lambda \mathrm{T})}\right]} \mathrm{d} \lambda=\mathrm{B} \lambda^{-4} \mathrm{~T} \mathrm{e}^{-\left(\frac{a}{\lambda \mathrm{T}}\right)} \mathrm{d} \lambda$ where $\mathrm{B}$ and a are constants which is Rayleigh-Jean's formula.

\subsection{Idea of Photons:}

The concept of Quantum of Radiation suggested by Max Planck was successfully applied by Einstein (Fig.3j) in the year 1905 to the photoelectric effect, in the year 1907 by Peter Debye to the theory of specific heats, in the year 1913 by Niels Bohr to the theory of atom and in the year 1922 to the scattering of x-rays. From these applications, it became clear that not only radiation is emitted or absorbed in discrete amounts of quanta but also the same quantum structure is retained by radiation while

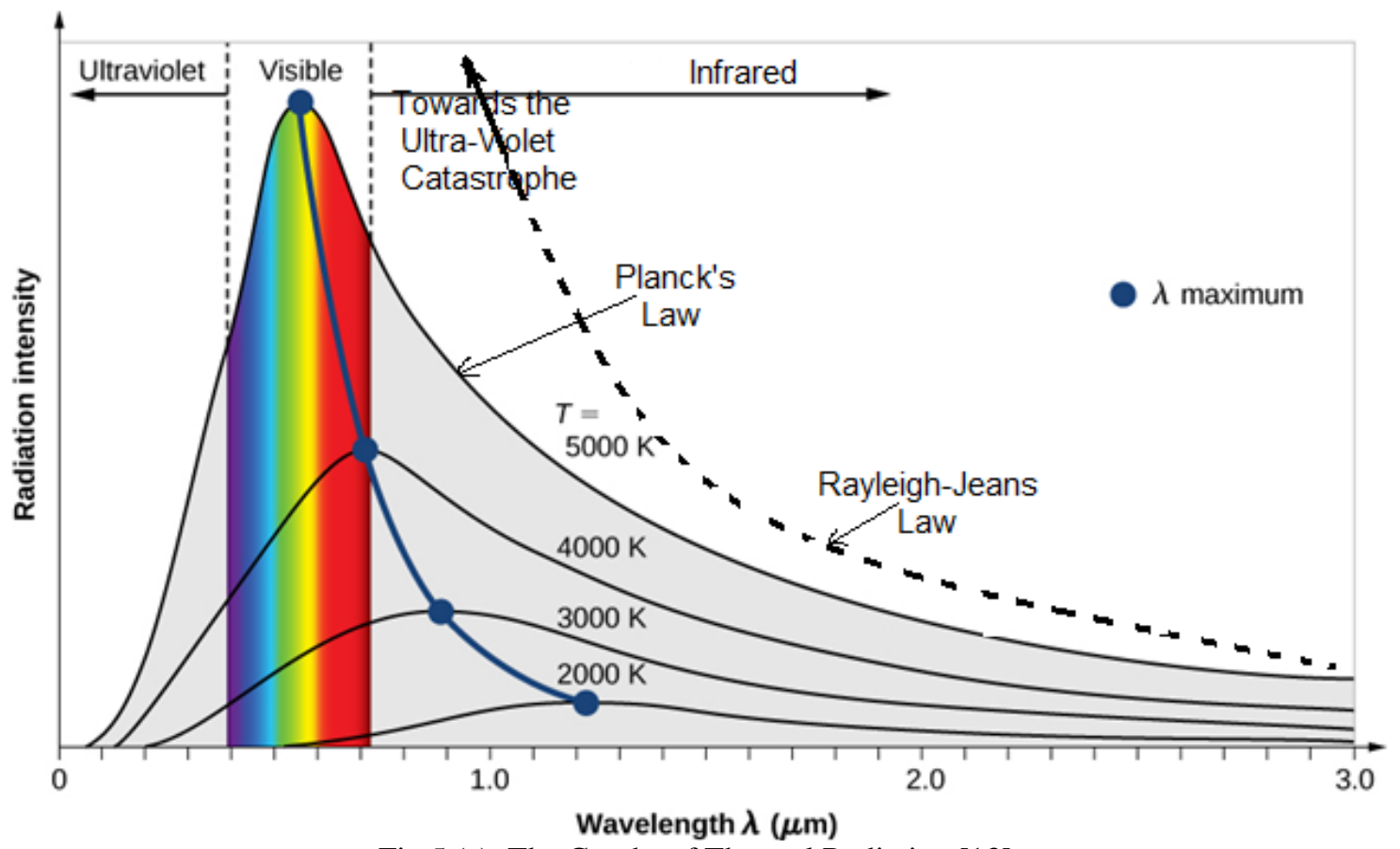

Fig.5 (a). The Graphs of Thermal Radiation [13]

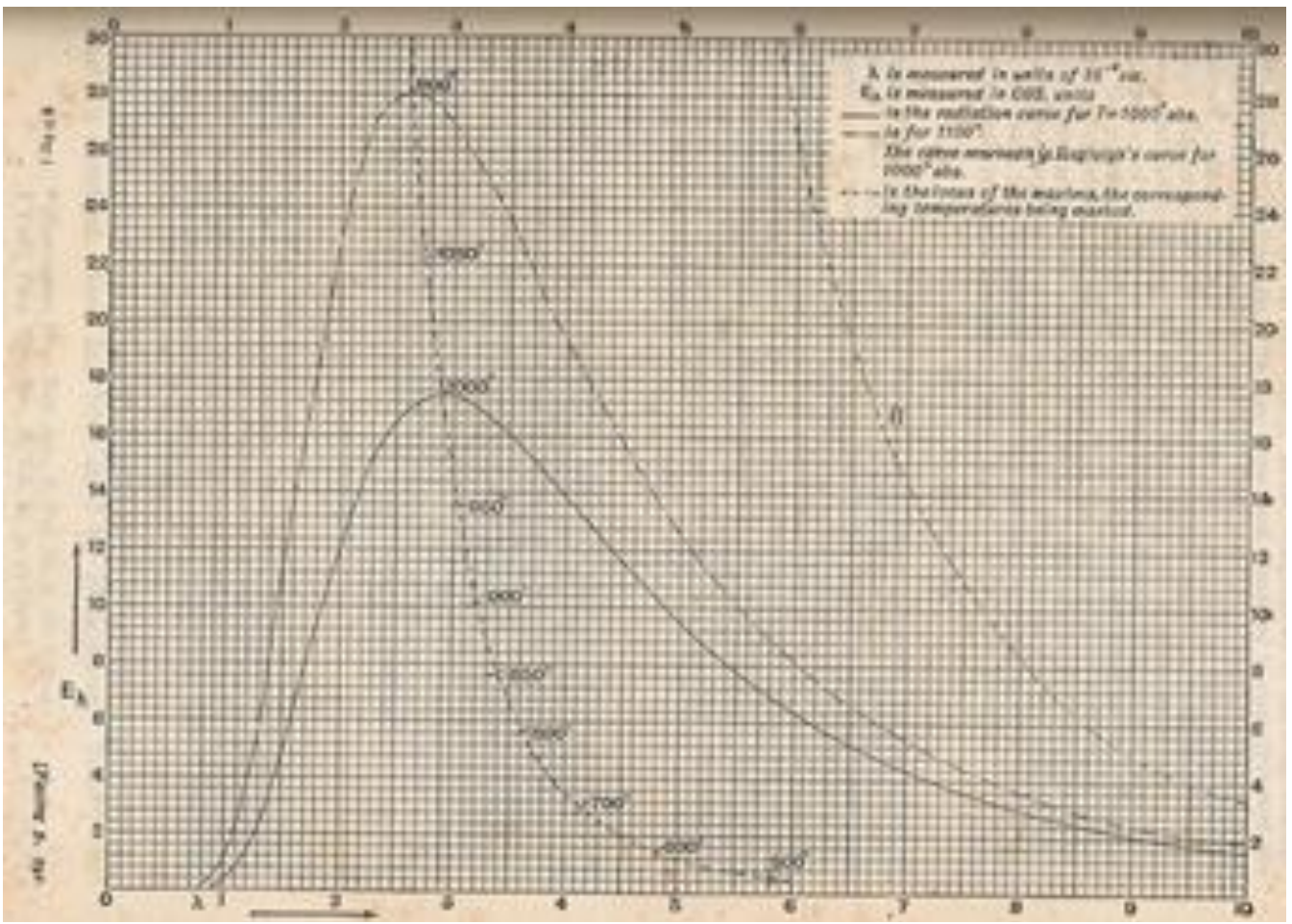

Fig.5(b) Graphs of Thermal Radiation on the background of a graph paper almost 80 year old [9] 


\section{International Advanced Research Journal in Science, Engineering and Technology}

travelling through space, very much like a shot fired from a gun. This is an extension of Planck's original hypothesis and according to it radiation is considered corpuscular in nature, made up of discrete quanta or photons, as they are now called, which are shot in space with velocity of light.

The corpuscular nature of radiation was verified by Einstein in his famous photoelectric effect for which he was awarded the Nobel Prize in 1921. If the electron is to be knocked out of a metal, simply energy of the quantum is not sufficient, but it should have material properties. For example, a man having enough energy to climb a coconut tree cannot get the coconut unless and until he personally climbs the tree and plucks it.

If radiation is corpuscular, then the question is, what is the difference between Matter and Radiation? The difference is that the rest mass of a material body is always finite and greater than zero whereas the rest mass of a photon is zero as shown below:

We have for a photon moving with velocity $\mathrm{v}$

$$
\begin{aligned}
& \mathrm{E}=\mathrm{h} v= \mathrm{m} \mathrm{c}^{2}=\frac{\mathrm{m}_{0} \mathrm{c}^{2}}{\sqrt{\left(1-\frac{\mathrm{v}^{2}}{c^{2}}\right)}} \\
& \therefore \frac{\mathrm{h} v}{\mathrm{~m}_{0}}=\frac{\mathrm{c}^{2}}{\sqrt{\left(1-\frac{\mathrm{v}^{2}}{c^{2}}\right)}}
\end{aligned}
$$

As $\mathrm{c}$ is constant, we have when $\mathrm{v}$ tends to $\mathrm{c}$, the quantity on the right tends to infinity in which case $\left(\frac{\mathrm{h} v}{\mathrm{~m}_{0}}\right)$ must also tend to infinity. This is possible if $\mathrm{m}_{0}$ tends to zero. If the rest mass is zero, then it means that there is no light quantum. The exact synthesis between matter and radiation is not simple but really complex.

\subsection{Wave Nature of Matter:}

\section{DE BROGLIE'S THEORY OF MATTER WAVES}

With the recognition of the quantum theory in the beginning of the twentieth century, physicists were obliged to admit a dual nature, wave and particle for the radiant energy. A similar situation arose with matter, when in the year 1924 a French physicist, Louis de Broglie (1892-1987) (Fig.4 a) put forward a bold suggestion that matter which is ordinarily made up of discrete particles (molecules, atoms, protons, electrons and the like) might exhibit wave-like properties under appropriate conditions. This means that matter like radiation has a dual nature.

De Broglie conceived the idea from "Nature loves symmetry" according to which the two fundamental forms of matter and energy, in which the nature manifests itself must be mutually symmetrical. Thgis is shown by Einstein in his famous Mass-Energy Equivalence. Since radiant energy has been shown to possess a dual nature - wave and particle, matter also must possess the same dual nature - particle and wave.

De Broglie asserted that these waves are generated in the motion of any body, whether a planet, a stone, a particle of dust or an electron. Like electromagnetic waves these waves are capable of propagation in vacuum and hence they are not mechanical waves. But they are produced in the motion of all bodies including those not charged electrically and hence they are not electromagnetic waves. Our ear responds to sound waves, which are mechanical waves of the longitudinal type. Our eyes respond to electromagnetic waves. As de Broglie waves do not belong to either class, we are not able to detect them.

When we talk of a wave, an important characteristic of which is its wavelength which de Broglie obtained by applying relativistic principles and quantum theory. There are many ways of arriving at it and I would like to present the one given by de Broglie in his Nobel lecture [3, p.244-256]

From the theory of quanta, energy $=\mathrm{h} x$ frequency where $\mathrm{h}$ is the Planck's constant

$$
\text { Or } \mathrm{W}=\mathrm{h} v
$$

where $\mathrm{W}$ is the energy a symbol used by de Broglie in his paper and $v$ the frequency.

In terms of rest mass $\mathrm{m}_{0}$

$$
\mathrm{h} v_{0}=\mathrm{m}_{0} \mathrm{c}^{2}
$$

This relation defines the frequency $\nu_{0}$ as a function of the rest mass $\mathrm{m}_{0}$ or inversely the quantity of movement (momentum) is a vector $\mathrm{p}$ given by

Hence we have

$$
\mathrm{p}=\frac{\mathrm{m}_{0} \mathrm{~V}}{\sqrt{\left(1-\beta^{2}\right)}} \text { where } \beta=\frac{\mathrm{v}}{\mathrm{c}}
$$

$$
\text { (p) }=\frac{\mathrm{m}_{0 \mathrm{v}}}{\sqrt{\left(1-\beta^{2}\right)}}=\frac{\mathrm{W} v}{\mathrm{c}^{2}}=\frac{\mathrm{h} v}{\mathrm{v}}=\frac{\mathrm{h}}{\lambda} \quad \ldots \ldots . . \mathrm{p} .249
$$

The quantity $\lambda$ is the distance between two consecutive peaks of the wave. That is the wavelength. Hence

$$
\lambda=\frac{\mathrm{h}}{\mathrm{p}}
$$

This is a fundamental relation of the theory.

In the Nobel lecture on p.251-2 de Broglie mentions and hinted as follows:

"A new mechanics must be developed which is to classical mechanics what wave optics to the geometrical optics" 


\section{International Advanced Research Journal in Science, Engineering and Technology}

ISO 3297:2007 Certified

Vol. 4, Issue 12, December 2017

This new mechanics has since been developed, thanks mainly to the fine work done by Schrodinger. It is based on wave propagation equations and strictly defines the evaluation in time of the wave associated with a corpuscle.

The two mechanics wave and quantum are equivalent from the mathematical point of view. As the Planck' constant $\mathrm{h}$ is involved in the de Broglie's formula for the wavelength, the de Broglie waves are essentially quantum in nature.

\subsection{Illustration:}

As a matter of interest, we may calculate the de Broglie wavelength particles given by V. Rydnik[10,p.71-2] As for a scientist like Einstein, the planet Earth and an electron are just particles, we may start with our Earth as a particle and end with an electron.

(a) For the planet, Earth

Mass, $\mathrm{m}=6 \times 10^{24} \mathrm{~kg}$

Orbital velocity, $\mathrm{v}=3 \times 10^{4} \frac{\mathrm{m}}{\mathrm{s}}$

$\therefore \quad \lambda=\frac{\mathrm{h}}{\mathrm{mv}}=\frac{6.6 \times 10^{-34}}{\left(6 \times 10^{24}\right)\left(3 \times 10^{4}\right)}=3.6 \times 10^{-63}$ meter.

This is fantastically small. No existing or foreseeable instruments could record anything that small.

(b) For a stone weighing 100 gram and travelling with a speed of, say one meter per second, we have

$$
\lambda=\frac{\mathrm{h}}{\mathrm{mv}}=\frac{6.6 \times 10^{-34}}{0.1 \times 1}=6.6 \times 10^{-33} \text { meter }
$$

This is also exceedingly small but much larger than that of the planet Earth in the previous case and a million million million times smaller than the atomic nucleus which itself is beyond the range of any microscope.

(c) For an electron moving in an electric field, the potential difference of which is 1 volt, acquires a velocity of $6 \mathrm{x}$ $10^{5} \frac{\mathrm{m}}{\mathrm{s}}$. The mass of an electron is about $10^{-30} \mathrm{~kg}$

$\therefore \quad \lambda=\frac{\mathrm{h}}{\mathrm{mv}}=\frac{6.6 \times 10^{-34}}{6 \times 10^{5} \times 10^{-30}}=10^{-9}$ meter

which is almost a nanometer and it corresponds to wavelength of $\mathrm{x}$-rays and can be detected.

Thus, in principle, we should be able to detect a de Broglie electron wave.

The experimental study of matter waves was done by Davisson, Germer, G.P. Thomson and many others. The could get a diffraction pattern of electrons when struck on a screen. Diffraction is essentially a wave phenomenon and the wavelength when measured is found to agree with theoretical results.

de Broglie's theory of matter waves, even though, was experimentally proved, there were lot of criticism in scientific circles regarding the authenticity of the theory. Even though de Broglie asserted that the motion is wave-like, he could not present an equation for the wave motion. It was something like knowing the brain of a man without seeing him. Two years later that is in 1926 physicists from all over the world arrived in Brussels at Solvay Congress. At this session, de Broglie's representation on the relation between waves and particles was totally and resoundingly rejected. For many years to come, a completely different representation of this relationship led the way. It was presented at the Congress by two young German physicists, Werner Heisenberg (1901-1976) (Fig.4 b) and Erwin Schrodinger (18871961)(Fig.4 c). Schrodinger from the then Austria.

\section{THE SCHRODINGER WAVE EQUATION}

Schrodinger in 1926 directly started with de Broglie's idea of matter waves and developed it into a rigorous mathematical theory which has received the name of "Wave Mechanics". How the idea was conceived by Schrodinger is mentioned by Michael Brooks [6, p.29]. Schrodinger worked out the mathematical implications of de Broglie's formula during a Christmas holiday in 1925. Leaving his wife in Zurich, Schrodinger took his mistress off to a chalet in the Swiss mountains. It was not unusual behavior for him- he and his wife seemed to come to several 'arrangements' through their marriage. Whatever went on, the trip was obviously inspiring. Schrodinger came back from the mountains in the new year (1926) with what is known as the Schrodinger wave equation.

The essential feature of the wave equation is the incorporation of the expression for the de Broglie wavelength into the general classical wave equation of d'Alembert. By this means a single equation which could describe both the motion of the particles and the propagation of waves was obtained. It is known as Schrodinger's fundamental wave equation which looks like this: 


\section{International Advanced Research Journal in Science, Engineering and Technology}

$$
\nabla^{2} \psi+\frac{8 \pi^{2} m}{\mathrm{~h}^{2}}(\mathrm{E}-\mathrm{V}) \psi=0
$$

Where $\nabla^{2}=\left(\frac{\partial^{2}}{\partial x^{2}}+\frac{\partial^{2}}{\partial y^{2}}+\frac{\partial^{2}}{\partial z^{2}}\right)$ is the Laplacian operator, $\mathrm{m}$ the mass of the particle, $\mathrm{h}$ the Planck's constant, $\mathrm{E}$ the total energy, $V$ the potential energy so that $(\mathrm{E}-\mathrm{V})$ will be the kinetic energy. $\psi$ denotes periodic changes which are responsible for the wave nature of the particles. The quantity $\psi$ is, in general, very complicated and the equation is universally the most fundamental. $\psi$ can be assigned the meaning of any event that takes place in nature. (5) is an equation with respect to space Another Schrodinger equation containing the time factor is:

$$
\frac{\mathrm{ih}}{2 \pi} \frac{\partial \psi}{\partial \mathrm{t}}=\frac{\mathrm{h}^{2}}{8 \pi^{2} \mathrm{~m}} \nabla^{2} \psi-\mathrm{V} \psi
$$

Equation (7) is unique among the equations of mathematical physics as it includes the imaginary quantity, $\mathrm{i}=\sqrt{-1}$. In general, the two equations (6) and (7) form the essence or backbone of the Wave Mechanics of Schrodinger.

What is of more interest and rather complicated is the interpretation of $\psi$. The interpretation was given by various physicists among whom the one given by the German physicist and mathematician, Max Born (1882-1970) (Fig.4d) is the most convincing. According to his interpretation, $\psi$ is a complex quantity and is called the "Probability Amplitude". The $\psi$ in the Schrodinger equations is such that the probability of finding the particle at a given point is proportional to the value $\psi^{2}$ at that point. I $\mathrm{f}$ the particle is to be found necessarily in a volume $(\mathrm{dxdy} \mathrm{dz})$, then the condition to be satisfied is:

$$
\iiint|\psi|^{2} \mathrm{dx} d \mathrm{y} d z=1
$$

A $\psi$ satisfying the condition given by equation (8) is known as "Normalized Wave Function"

Heisenberg using Matrix Mechanics independently worked out the theory and arrived at the same results of Schrodinger. The mechanics developed by Heisenberg is known as New Quantum Mechanics.

\section{THE PRINCIPLE OF INDETERMINACY OR THE UNCERTAINTY PRINCIPLE}

\subsection{The Uncertainty Relations:}

From the probability interpretation of the wave function, Heisenberg in 1927, was able to draw a very interesting conclusion of far reaching importance known as 'Principle of Indeterminacy' or the 'Uncertainty Principle' This has been given different names by different authors. Richtmyer [8, p.299] has given its original name before it is translated. That is, "Principle of Unbeslimmtheit". This has been variously translated as indeterminacy, indefiniteness or uncertainty. Sir Edmund Whittaker [12, p.143] has mentioned the name "Principle of Imperfect Specification".

According to wave mechanics, it is the wave associated with the particle that represents or symbolizes all that we know about the particle. By saying "All that we know about the particle" means its position and velocity. But, according to Heisenberg's uncertainty principle, it is impossible to determine simultaneously both position and velocity of the particle with accuracy.

As for an example, we may try to find the position and velocity of a particle accurately. In order to see an object clearly, the wavelength of the illuminated light must be less than the dimensions of the object. We shall take two different particles, a dust particle and an electron. In the case of the dust particle, we can illuminate it by ordinary light photons and in the case of electrons we may use gamma ray photons having smaller wavelengths.

Assuming both particles are at rest, we find that in the case of the dust particle, it remains in its position and is not affected at all, whereas in the case of the electron, as soon as it is illuminated by the gamma rays, forget any change occurring, the electron is simply knocked right out of the picture. This is because of the greater momenta of the gamma ray photons and hence the position of the electron cannot be determined accurately.

If the particles are already in motion. Assuming the dust particle to be of a micron $\left(10^{-6} \mathrm{~m}\right)$ size, of density about $10^{4}$ $\frac{\mathrm{kg}}{\mathrm{m}^{3}}$ and let its velocity be 1 micro meter per second, then its mass will be $10^{-14} \mathrm{~kg}$ and momentum $10^{-20} \mathrm{~kg}$. meter per second. But, the momenta of photons of visible light is only $10^{-27} *$ which is tens of millions of times less than the momentum of the dust particle. Thus the photon impacts will not make any effect on the dust particle.

*Frequency of visible light is $5 \times 10^{14} \mathrm{~Hz}$, the. Thus momentum of photons of visible light is

$$
\frac{\mathrm{h} v}{\mathrm{c}}=\frac{6 \times 10^{-34} \times 5 \times 10^{14}}{3 \times 10^{14}}=10^{-27}
$$

Now, coming to the electron, we may even assume that it moves with the velocity of light. Taking approximate values, the momentum of the electron is $\mathrm{mv} \approx\left(10^{-30}\right)\left(10^{8}\right)=10^{-22} \frac{\mathrm{kg} \mathrm{m}}{\mathrm{s}}$. But the momentum of high frequency gamma ray photon used to illuminate the electron is of the order of about $10^{20}$ which is much greater than that of the 


\section{International Advanced Research Journal in Science, Engineering and Technology}

electron. So when a photon hits an electron, the situation is similar to a speeding locomotive shunting a slow moving wagon. Thus we cannot determine the velocity of the electron

Heisenberg who was the first to realize these consequences, expressed them mathematically by means of the following equation known as the uncertainty relation.

$$
\Delta x \times \Delta \mathrm{v} \approx \frac{\mathrm{h}}{\mathrm{m}},
$$

where $\Delta x$ and $\Delta \mathrm{v}$ are the respective uncertainties (or possible errors) involved in the simultaneous determination of the position and velocity of a particle and is approximately equal to the Planck's constant divided by the mass of the particle. The relation can also be expressed in terms energy, $\mathrm{E}$ and time, $\mathrm{t}$ in which case the relation can be written as:

$$
\Delta \mathrm{E} \times \Delta \mathrm{t} \approx \frac{h}{2 \pi}=10^{-34} \text { Joule second }
$$

The equation is such that if any one of the uncertainties tends to zero, then the uncertainty in the other must tend to infinity.

The equation (9) is generally written in terms of the position, $x$ and momentum, $p$ in which case it takes the form:

$$
\Delta x \times \Delta \mathrm{p}_{x} \approx \frac{\mathrm{h}}{2 \pi}
$$

where $\Delta \mathrm{p}_{x}$ is the momentum in the $\mathrm{x}$-direction.

In the classical and exhaustive book by Ritchmyer [8] followed by a large number of professors and students of yester years (Well !, I am one of them), the equation (11) is written as:

$$
\Delta x \times \Delta \mathrm{p}_{x} \geq \frac{\hbar}{2} \ldots \text { [Eq. 11.17, p.297] .... }
$$

The right hand side is the reduced Planck's constant, $\hbar$ divided by 2 . That is, $\frac{h}{4 \pi}$. Except that the value on the right hand side is still made smaller, there is no change otherwise.

The essence of the uncertainty principle is that we cannot determine simultaneously both the position and momentum of an electron with same accuracy. This sets an upper limit in the purity of an observation. One quantity has to be sacrificed for want of the other. One cannot eat the cake and also have it. I have introduced for the benefit of readers and for better understanding a Heisenberg Indeterminacy Balance (Fig.6)

We may consider a macroscopic and microscopic body such as a bullet and an electron respectively and worked out the uncertainties by taking specific values.

i)A bullet weighing 50 gram is shot with a speed of 300 meter per second and accurate to $0.01 \%$. The fundamental accuracy in the position of the bullet can be calculated as follows:

Mass of the bullet, $\mathrm{m}=50$ gram $=0.05 \mathrm{~kg}$

Momentum of the bullet, $\mathrm{p}=$ mass $\mathrm{x}$ velocity $=0.05 \times 300=15 \frac{\mathrm{kg}}{\mathrm{s}}$

$$
\Delta \mathrm{p}=(0.0001)(15)=1.5 \times 10^{-3} \frac{\mathrm{kg} \cdot \mathrm{m}}{\mathrm{s}} \text {. }
$$

We have from equation (11), $\Delta x=\frac{\hbar}{1.5 \times 10^{-3}}=\frac{10^{-34}}{1.5 \times 10^{-3}}=0.67 \times 10^{-31}=6.7 \times 10^{-32}$ meter.

where we have substituted the approximate value of the reduced Planck's constant, $\hbar$

But the uncertainty $\Delta x$ obtained for the position is beyond the possibility of any measurement. (The diameter of the nucleus of an atom is of the order $10^{-15}$ meter, whereas $10^{-32}$ is very much smaller)

ii)Now, taking an electron having the same speed and accuracy as the bullet and mass approximated to $10^{-30} \mathrm{~kg}$ for

simplicity, then we have fr the momentum,

$$
\mathrm{p}=\mathrm{m} \mathrm{v} \quad 10^{-30} \times 300=3 \times 10^{-28} \frac{\mathrm{kg} \cdot \mathrm{m}}{\mathrm{s}}
$$

The uncertainty in the momentum is $0.001 \%$ of this and hence

$\begin{aligned} & \Delta \mathrm{p}=0.0001 \mathrm{x} 3 \mathrm{x} \\ & \text { Agin applying in equation (11), we get for } \Delta \mathrm{x}\end{aligned}$

$$
\Delta \mathrm{p}=0.0001 \times 3 \times 10^{-28}=3 \times 10^{-32}
$$

$$
\Delta \mathrm{x}=\frac{\hbar}{3 \times 10^{-32}}=0.3 \times\left(10^{-34}\right)\left(10^{32}\right)=0.3 \times 10^{-2} \text { meter }=0.3 \mathrm{~cm}
$$

I have applied this principle to human life as well. The human body as a whole is a huge macroscopic system built up with innumerable microscopic components made to work in unison in such a way that even any best fabricator on Earth cannot imagine to operate. The uncertainty takes place almost daily and remains unknown. The minimum energy required to activate our human mind is just $10^{-11}$ Joule and the 


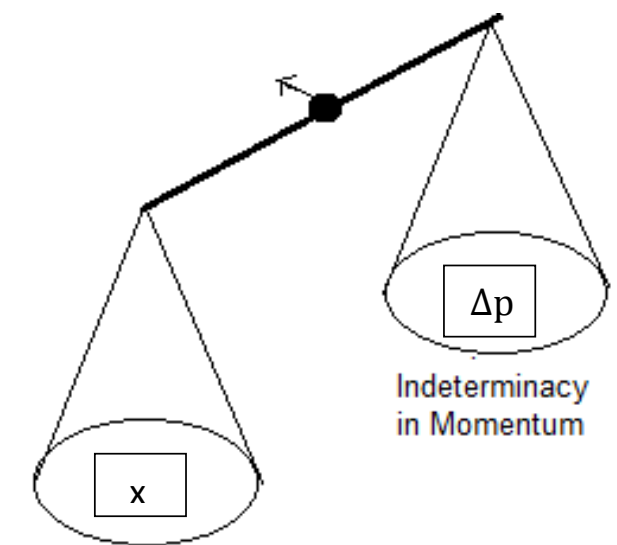

Determinacy in Position
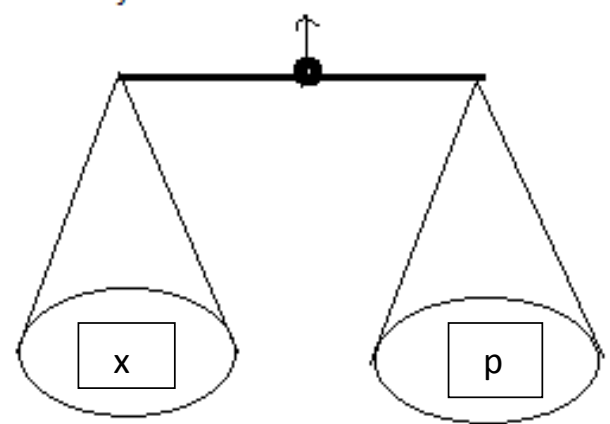

Determinacy in Position Determinacy in Momentum

(This is an absolute Impossibility)

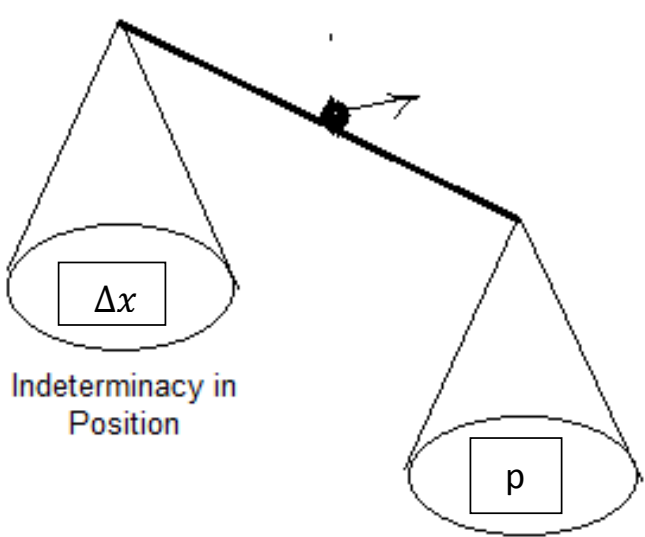

Determinacy in Momentum

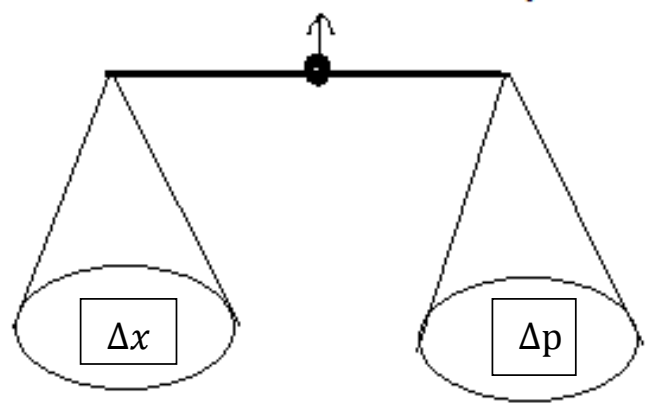

Indeterminacy in both Position and Momentum This is the Uncertainty Principle

The product of which is

Fig. The Heisenberg's Indeterminacy Balance (Illustration by author)

time required for this activation can be taken as the time required for a fast moving particle to pass through the nucleus of an atom which is $10^{-23}$ second. These are the uncertainties in the thoughts of the human mind. Thus the action of the human mind is $\left(10^{-11}\right)\left(10^{-23}\right)=10^{-34} \mathrm{Js}$ which is almost equal to the reduced Planck's constant $\frac{h}{2 \pi}=1.055 \times 10^{-34} \mathrm{Js}$.

As Heisenberg says that when you do not know your position, you do not know your motion and when you know your motion, you do not know your position. This uncertainty can be applied for the time at which a man sleeps. If somebody says that he slept at 10 PM by looking at a watch just before sleeping. Well! When he has looked at the watch he was not asleep. Now, when he fell asleep, at what exact time he fell asleep is not known. Here comes the Uncertainty of Heisenberg. When he is asleep, he is not knowing the time and when he knew the time, he was not asleep. I think Heisenberg also did not think about this. In the modern advancement in medicine, some neurologist, by some test of his brain condition can find out the exact time of his sleep. The same is the case with a passenger in an airconditioned coach with all windows closed. He is not knowing the speed of the train nor does he know the railway station for which when he comes out, several places have been already covered. It is difficult to apply the principle in such macroscopic systems, but such uncertainties exist in all walks of life. If somebody says that a meeting will be held at $11 \mathrm{AM}$ and invites people to attend. Now, apart from people coming earlier or late, there are uncertainties in the time shown by the clocks of people attending the meeting and also in the clock of the person who called for the meeting

Even the Earth is not a perfect clock. It slows down during its rotation. The discrepancies in astronomy and astrology are due to such uncertainties. The failures in astrology are due to the discrepancies in locating planetary positions.

David Bohm [2,p.169-70] has connected the uncertainty principle with certain aspects of our thought processes. He states that if a person tries to observe what he is thinking about at the very moment that he is reflecting on a particular subject, it is generally agreed that he introduces unpredictable and uncontrollable changes in the way his thoughts proceed thereafter. The thought processes and quantum systems are analogous controlled by the principle of uncertainty.

Now, the problem is as to why these uncertainties exist? What is to be blamed, the measuring instrument or the electron? Now-a-days we have the most modern and accurate instruments which can measure with very high precision and hence the blame goes to the electron. But, the results with such instruments still showed uncertainties. Thus, it 


\section{International Advanced Research Journal in Science, Engineering and Technology}

ISO 3297:2007 Certified

Vol. 4, Issue 12, December 2017

appears, that both are to be blamed, but only half and half. The truth of the matter is that Heisenberg's relation exhibits the 'guilt' of both the instrument and electron. The movement or the characteristics associated with the electron is so universal and beyond the creation of any human ingenuity, we can blame the instrument. "The failure of the instrument is then due precisely to the wave property of the electron.

The principle of uncertainty governs any measurement macroscopic or microscopic that is carried out in the universe. The principle must be regarded not as a complete surrender of determinism in Physics for that matter in any of its applied branches, but as a further refinement in the right direction showing that the deterministic conception of nature is neither complete nor universal.

An important point to be stressed is that by saying an electron wave is not that it is an aircraft with electron as a passenger. The electron is not a wave. Neither the wave contributes an electron. It is the law of mechanics which makes the motion of electron wave-like in character. The moment the electron comes to rest, the wave nature abruptly disappears. The famous English physicist and grandson of Charles Darwin, Charles Galton Darwin proposed calling the de Broglie matter wave as neither a wave nor a particle, but appropriately "Wavicle” [8 . p299]

This is what J.B. Rajam [7, p.457] has to say in this regard of dualistic nature of both matter and radiation. He says that "the particle and wave aspect are strictly complementary" in the sense that although the spatial distribution of particles can only be predicted by taking into consideration the concept of waves, yet when one tries to define precisely the wave aspect, the particle aspect recedes into the background and becomes indefinite being a density distribution as per some of the interpretation of the wave function. Thus, in reality, whether matter or radiation is made up of a subtle and almost indefinable fusion of two antagonistic and complementary factors, the continuous wave and the discontinuous particle. It is a discontinuous continuity or a continuous discontinuity and hence not a simple but complex unity.

An eminent author, Simon Singh [11,p.492] writes in his famous book, Big Bang regarding uncertainty principle as follows: "Although cause and effect is a valid principle in the macroscopic world, it is the so-called uncertainty principle that rules the sub-microscopic quantum domain. This principle dictates that events can happen spontaneously, which has been shown to be the case experimentally. It also allows matter to appear from nowhere, even if only temporarily; At the everyday level, the world seems deterministic and the laws of conservation hold true, but at the microscopic level determinism and conservation can both be violated"

The quantum physicist, Michael Brooks [6, p.54 \&72] and author of the popular book, 'The Big Questions' mentions that Heisenberg's uncertainty principle is a fundamental characteristic of quantum theory and further says that the strong force in Quantum Chromo Dynamics (QCD), has its roots in the uncertainty principle of quantum mechanics. The principle even applies to empty space which cannot have exactly zero energy. As a result, empty space has a fluctuating but finite amount of energy.

Edwin C. Kemble [5, p.76-77], professor of Physics at Harvard University in his famous book on Quantum Mechanics mentions that the uncertainty principle has lot to do with the hypotheses of 'determinism' and 'indeterminism' and aroused a universal interest among physicists and philosophers. Classical determinism is the doctrine that, if the present state of an isolated system (eventually the universe) were known, the future behavior of the system could be exactly fortold by a sufficiently expert mathematician. This view is compatible with Quantum Mechanics, which not only denies the validity of the hypothetical calculation but through the principle of uncertainty asserts that the information required by the super mathematician is both unobtainable and meaningless in terms of operations which we can perform, even in principle. Although Quantum Mechanics is thus essentially indeterministic, a modicum of determinism remains in the fact that the $\psi$ function of an isolated system is uniquely determined for all time by its initial form. Indeterminism comes into the scheme in the relation before the wave function and the results of individual experiment, or measurement. As measurements always imply interaction of the system under observation with an external apparatus, it is possible to argue that the root of the indeterministic form of Quantum Mechanics lies in the apparently inevitable division of the universe into "Observed" and "Observer"

Physics Part-II by Resnick and Halliday, an under graduate level book [p.1213] mentions that if you toss a coin, you can see either the face or the tail and not both.

The de Broglie's theory of matter waves and the Heisenberg's uncertainty principle are the apples of the same tree with the roots of Quantum Theory - Author. 


\section{International Advanced Research Journal in Science, Engineering and Technology}

ISO 3297:2007 Certified

Vol. 4, Issue 12, December 2017

\section{CONCLUSION}

As a conclusion, I would like to mention that, in what way the introduction of the Planck's constant revolutionalized the entire Physics, the same manner, the idea of matter waves of de Broglie created an altogether a different form of Mechanics, viz. Wave Mechanics with which the behavior of micro particles could be studied better. The uncertainty principle is applied in the research of particle physics as in the Standard Model.

\section{ACKNOWLEDGEMENT}

The main reference in the paper is from Atomic Physics by J.B. Rajam [7]. Rajam is an eminent author of Atomic Physics in the 50's. It is a widely read and followed text book at the graduate and post-graduate level. The book has nearly 1200 pages with Author Index, Subject Index and each chapter reference given separately. The salient feature of the book is that it begins with an exhaustive Foreword by Prof. Louis de Broglie (Nobel Laureate), Institut de France, Academic des sciences. De Broglie has tremendous appreciation for the book and has congratulated the author, J.B. Rajam in the great preparation of the book.

I hereby acknowledge J. B. Rajam for the matter, language, expression and general presentation.

\section{REFERENCES}

(Page Nos. in the references appear in the text)

[1] Bergmann P.G."Basic Theorties of Physics-Heat and Quanta", Dover Publications, N.Y.1962.

[2] Bohm David. "Quantum Theory", Asia Publishing House, Indian Edition 1960

[3] De Broglie Louis Victor, "The Wave Nature of Electrons" Dec. 12, 1929.

[4] Heisenberg. "Quantum Theory”.'Dover Publications Inc. 1930

[5] Kemble Edwin C, "The Fundamental Principles of Quantum Mechanics with Elementary Applications"Dover Publications Inc. NY.1958

[6] Michael Brooks. "The Big Questions- Physics". Metro Books New York. No date appearing

[7] Rajam J.B. "Atomic Physics". S. Chand \& Co. $4^{\text {th }}$ Ed. 1958

[8] Richtmyer, Kennard and Cooper. "Introduction to Modern Physics".Mc-Graw Hill Book Co. $6^{\text {th }}$ Ed.1969.

[9] Roberts J.K."Heat and Thermodynamics". Blackie and Son Ltd. N.Y. ${ }^{\text {rd }}$ Ed. Reprint 1943.

[10]Rydnik. "ABC's of Quantum Mechanics". Peace Publishers Moscow. No date appearing

[11]Simon Singh. "Big Bang-The Origin of the Universe". Harper Perennial. 2004

[12]Whittaker Edmund. "From Euclid to Eddington". Dover Publications. N.Y.1958

[13]Wikipedia

\section{BIOGRAPHY}

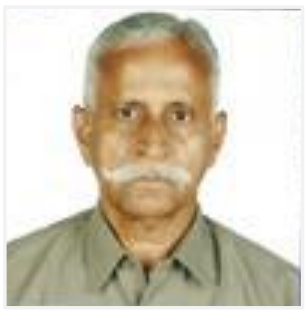

*Dr.(Prof.) V.C.A. Nair (b.15th Aug. 1939) is an Educational Physicist, Counselor, Research Guide and Consultant. He did his Masters in Physics from Mumbai University, India and Ph.D. from JJT University, Rajasthan also in India He is a Research Guide and distinguished alumni of JJT University. He is also a Chancellor designated Resource Person in the area of Physics in the University. He has to his credit over 4 decades of teaching Applied Physics in eminent Polytechnics in Mumbai and having taught nearly 16,000 students since 1965. He has published a number of research papers in Physics and Geophysics in International and UGC recognized Journals some of which can be seen in the net 'Google Search' when the name of the author is typed in that style. He is a Life Member of Indian Society for Technical Education which is an all India body. He had been to USA a number of times and visited eminent Universities such as Stanford, Harvard, MIT, University of California both at Berkeley and Los Angeles and University of San Francisco. At present Dr. Nair is a Research Guide for Physics at JJT University, Rajasthan-333001, India . He is a member of the Editorial Board of this Journal. His Ph.D. Thesis is in Geophysics and he is working on topics such as Tides, Clouds, Global Warming and Climate Change. - Editor. *nairvca39@gmail.com. 